\title{
Retour d'expérience de deux structures d'urgences de centres hospitaliers généraux pendant la crise de la Covid-19
}

\section{Feedbacks from Two General Hospital Emergency Departments during COVID-19 Crisis}

\author{
M. Noizet $\cdot$ Y. Gottwalles $\cdot$ S. Nguyen $\cdot$ E. Thibaud $\cdot$ F. Labrosse $\cdot$ N. Delmas $\cdot$ E. Vilbois $\cdot$ J. Marchetto $\cdot$ \\ S. Heymonet
}

Reçu le 16 juillet 2020 ; accepté le 4 août 2020

(C) SFMU et Lavoisier SAS 2020

Résumé La Covid-19 s'est abattue sur l'Alsace en quelques jours, mettant à mal les hôpitaux de Mulhouse et de Colmar. Bien que proches, des différences notables existent au sein de ces deux structures en termes architecturaux et de ressources humaines. Comment cette vague a-t-elle modifié les organisations de chacun ? Quelles alternatives ont pu être mises en place? Après la présentation de chaque site sont analysées les adaptations nécessaires afin d'absorber les flux de patients et leur proposer une prise en charge décente malgré des conditions parfois très dégradées. Nouvelles filières, modifications organisationnelles, renforts de personnel en interne puis en externe, augmentation capacitaire nette, recherches de solutions alternatives aux voies classiques d'approvisionnement des matériels... ont été une partie de la solution. Malgré les alertes du terrain, l'action nationale est restée en décalage de compréhension de la gravité de la situation locale, avec des recommandations et des actions soit inapplicables, soit trop tardives. Les établissements et professionnels de santé ont eu un sentiment d'isolement, renforcé par l'absence de représentant des tutelles et notamment de l'Agence régionale de santé sur le terrain. Deux éléments majeurs peuvent être mis en avant : une cohésion majeure de l'ensemble du centre hospitalier avec travail d'équipe et de terrain entre soignants et direction, et une implication de l'ensemble des acteurs de la médecine d'urgence (public, privé, libéraux, service départe-

\footnotetext{
M. Noizet $(\varangle) \cdot$ S. Nguyen $\cdot$ F. Labrosse $\cdot$ E. Vilbois Structure d'accueil des urgences, groupe hospitalier Mulhouse et Sud Alsace, 20, avenue du Docteur-René-Laennec, F-68100 Mulhouse, France

e-mail : marc.noizet@ghrmsa.fr

Y. Gottwalles · E. Thibaud · N. Delmas · J. Marchetto

S. Heymonet

Structure d'accueil des urgences, pôle urgences Pasteur, Hôpitaux civils de Colmar, 39, avenue de la Liberté, F-68000 Colmar, France
}

mental d'incendie et de secours) ; ils ont été les déterminants dans notre capacité à faire face à cet événement inédit.

Mots clés Covid-19 · Pandémie · Retour d'expérience · Organisation

\begin{abstract}
COVID-19 fell down on Alsace in few days, putting a strain on both hospitals of Mulhouse and Colmar. Although close, there are significant differences between these two structures in terms of architecture and human resources. How has this wave modified both organizations? After the presentation of each site, we analyzed the necessary adaptations made to absorb the flow of patients and offer them decent care despite sometimes very deteriorating conditions. New pathways, organization modifications, internal then external staff reinforcements, net capacity increase, search for alternative solutions to traditional equipment supplies routes, etc. have been a part of the solution. Despite the alerts from the ground, national action remained out of step with understanding of the seriousness of the local situation, with recommendations and actions either inapplicable or too late to implement. There was a strong feeling of isolation among health care organizations and professionals, reinforced by the absence of representatives from health institutions and particularly from the regional health agency in the field. Two main elements can be highlighted: a strong cohesion of the whole hospital center through teamwork and groundwork between the caregivers and the management, as well as the implication of all the actors of the emergency medicine (public staff, private staff, liberal staff, departmental fire, and rescue service); they were the determining factors in our capability to face this unprecedented event.
\end{abstract}

Keywords COVID-19 · Pandemic · Feedbacks · Organization 


\section{Introduction}

En février 2020, le département du Haut-Rhin a fait face à une crise sanitaire majeure conséquence de la diffusion fulgurante de l'épidémie de Covid-19. Cette épidémie s'est développée à partir d'un rassemblement cultuel qui a eu lieu en périphérie de Mulhouse du 17 au 23 février. Les centres hospitaliers de Mulhouse, puis de Colmar, de par leur proximité immédiate, ont été impactés avec une violence extrême, avec nécessité de modification organisationnelle majeure, d'adaptabilité et de remise en question permanente. Au-delà, c'est le système de santé français qui a dû faire face à la vague de Covid-19, avec très peu d'annonces fiables, très peu de connaissances sur un virus connu que depuis quelques mois et des données validées sporadiques s'appuyant sur l'expérience chinoise. Les connaissances se sont faites au fil de l'eau, de jour en jour, parfois avec des contradictions, toujours avec des incertitudes, mais avec une rapidité d'étranglement des centres hospitaliers majeurs.

\section{Description des structures d'urgences}

Le Groupement hospitalier de la région Mulhouse et Sud Alsace (GHRMSA) est un établissement de 1250 lits d'hospitalisation complète, avec une structure d'urgences (SU) associant trois sites d'accueil d'urgences totalisant 120000 passages annuels, le site de Mulhouse accueillant 55000 patients par an.

La SU de Mulhouse est un service qui a traversé une crise profonde en 2019 avec pour conséquence majeure le départ de 17 médecins. C'est un service d'une grande fragilité qui avait débuté un processus de reconstruction après le recrutement extérieur d'un nouveau chef de service. Ce service présente alors au début de la crise certaines caractéristiques :

- une équipe médicale s'appuyant sur le remplacement ou les renforts extérieurs pour les deux tiers de ses besoins de fonctionnement ;

- une équipe paramédicale robuste avec un encadrement dynamique ;

- un SAMU-Centre 15 ayant investi de nouveaux locaux en 2018 et ayant gardé opérationnelle son ancienne salle de régulation avec vocation à servir de salle de régulation de crise ;

- un service d'accueil des urgences (SAU) exigu, avec une architecture inadaptée au niveau d'activité ;

- l'absence de plan ou procédure organisationnelle dédiée à adapter le SAU à un accueil de patients dans le cadre d'un afflux épidémique ou d'un risque nucléaire, radiologique, biologique, chimique ou explosif (NRBCe).

Situés à un peu plus de $40 \mathrm{~km}$ au nord de Mulhouse, les Hôpitaux civils de Colmar (HCC) constituent un centre hospitalier de 930 lits d'hospitalisation conventionnelle,
2730 paramédicaux et 324 médecins, avec un SU adultes accueillant 50000 primopassages par an. Plusieurs particularités s'attachent à ce service :

- une réhabilitation immobilière complète il y a moins de deux ans, avec accès valides/brancards distincts, des circuits médecine/traumatologie distincts, un circuit médecine libérale (ML), un total de 22 boxes d'examen ( 6 en médecine, 7 en traumatologie, 2 en salle d'accueil des urgences vitales (SAUV), 3 en salle tiède, 1 en salle de plâtre, 1 en salle de suture, 1 en ML, 1 en ophtalmologie), une zone de transit de 7 brancards et 4 piétons, une unité d'hospitalisation de courte durée (UHCD) de 15 lits, 1 zone de lits de 72 heures de 3 places;

- une équipe médicale de plus de 30 praticiens, à l'effectif cible ;

- une équipe paramédicale sans poste vacant, et un encadrement assuré par 2 référents d'unité et une cadre de santé ;

- un centre de référence NRBCe, avec des équipes formées par l'organisation biannuelle d'exercices grandeur nature pour accueil de radiocontaminés; de fait, une habitude de travailler en tenue intégrale, avec des zones sales/propres définies, et surtout un stock surdimensionné non seulement pour le centre hospitalier, mais également pour une SU de cette taille ;

- le chef de pôle et le chef de service assurent une activité clinique prédominante.

Tous les marqueurs semblaient se trouver au vert à Colmar face à une éventuelle pandémie : locaux opérationnels, équipes au complet, matériaux et stocks, habitude de travail en conditions de type NRBCe.

\section{Chronologie des faits}

Le 24 février, le directeur général de l'Organisation mondiale de la santé, Tedros Adhanom Ghebreyesus, a appelé le monde à se préparer à une "éventuelle pandémie » du nouveau coronavirus, en jugeant «très préoccupante [...] l'augmentation soudaine " de nouveaux cas en Italie, en Corée du Sud et en Iran [1]. La Lombardie et la Vénétie, proches d'un peu plus de $300 \mathrm{~km}$ du Haut-Rhin, ont décidé du confinement de plusieurs de leurs villes devant une augmentation importante du nombre de nouveaux cas de patients infectés. La proximité de ces régions et le partage de flux de population avec le Haut-Rhin ont amené une augmentation du nombre d'appels au SAMU-Centre 15. Nos deux SU ont mis en place un circuit d'accueil spécifique des cas suspects aux urgences. Les HCC ont dès lors mis en place une cellule de crise quotidienne.

Le 26 février, le CHU de Strasbourg, seul site de prélèvement et d'analyse des RT-PCR (reverse transcriptase polymerase chain reaction) Covid, a demandé au GHRMSA 
d'organiser un site de prélèvement devant une demande en provenance de la zone de Mulhouse devenue trop importante. Ce centre rapidement mis en place était opérationnel sept jours sur sept dès le 28 février.

Le $1^{\mathrm{er}}$ mars, l'enquête épidémiologique a permis d'identifier deux familles du Haut-Rhin ayant plusieurs cas avérés et ayant participé à un rassemblement évangélique de 2000 à 3000 personnes, dans la semaine du 17 au 23 février. Le niveau d'activité du SAMU-Centre 15 était à deux fois sa moyenne quotidienne.

Le 2 mars, les deux SU ont mis en place le port systématique des équipements de protection individuelle (EPI : masques FFP2, lunettes ou équivalent, surblouses) sur les postes de première ligne, infirmier d'organisation de l'accueil (IOA) et Smur, avant tout interrogatoire. Le GHRMSA a mis en place une cellule de crise bihebdomadaire et a décidé l'ouverture d'un secteur dédié de médecine unité Covid de 14 lits. Le SAMU-Centre 15 était renforcé en personnel.

Le 3 mars, l'annonce du directeur général de la santé, lors de sa conférence de presse quotidienne médiatisée, de l'identification de la cause des cas Covid + du département du Haut-Rhin comme étant liée au rassemblement évangélique a provoqué un afflux d'appels amenant le niveau d'appels à trois fois la moyenne quotidienne [2].

Le 4 mars, le SAMU-Centre 15 a rencontré une saturation de ses lignes suivie d'une panne de son infrastructure téléphonique et a ouvert sa salle de régulation médicale de crise, faisant appel à des médecins extérieurs, étudiants, secrétaires. Simultanément, nous avons mis en place le délestage de certains appels Covid vers les Samu 67 et 54. Devant le nombre de patients symptomatiques, il a été décidé l'arrêt des prélèvements des patients symptomatiques sans critère de gravité. Seuls les patients hospitalisés bénéficiaient d'un prélèvement en opposition au discours de santé publique national.

Le 7 mars, l'activité du SAMU-Centre 15 a été multipliée par quatre et a nécessité l'ouverture d'une nouvelle salle de délestage au CIP (centre d'information du public) de la préfecture puis au Centre opérationel départemental d'incendie et de secours (CODIS) 68. Le GHRMSA a déclenché son plan blanc et déprogrammé toutes ses activités médicales et chirurgicales.

La semaine qui a suivi a été marquée par une forte augmentation de l'activité d'accueil hospitalier de cas Covid (Fig. 1), dont une part importante nécessitait une hospitalisation du fait de leur oxygénodépendance, les hospitalisations en réanimation de patients nécessitant une assistance ventilatoire étaient de plus en plus fréquentes (Fig. 2).

Le 9 mars, la SU des HCC a dû faire face à un afflux d'admissions de cas Covid-19, cette date correspondant à un point de rupture, nous avons basculé l'organisation habituelle et consacré les trois quarts des surfaces de la SU à la prise en charge exclusive de patients Covid-19. Le plan

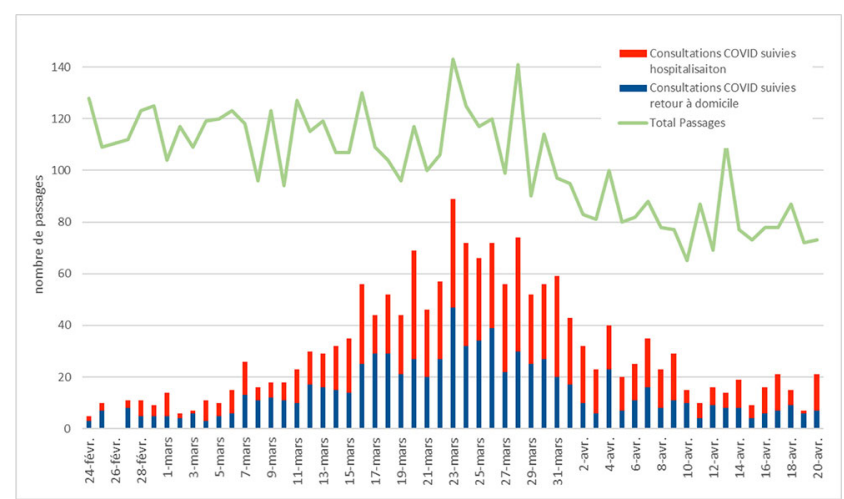

Fig. 1 Activité du service d'accueil des urgences du Groupement hospitalier de la région de Mulhouse et Sud Alsace (GHRMSA)

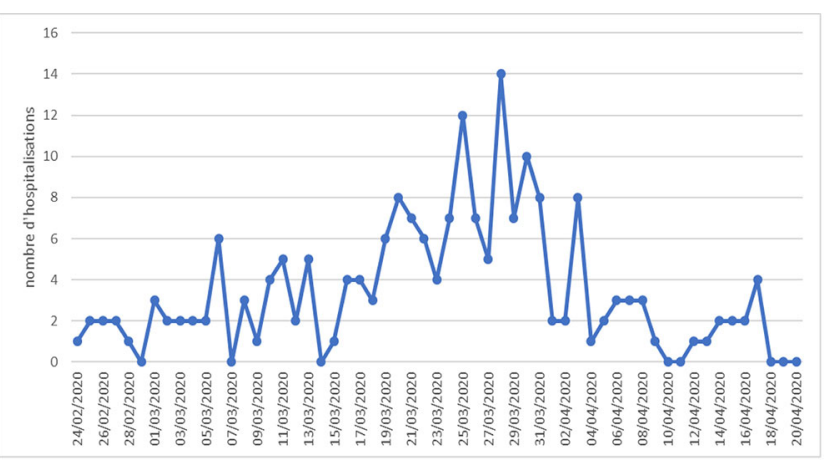

Fig. 2 Nombre d'hospitalisations en réanimation à partir du service d'accueil des urgences du Groupement hospitalier de la région de Mulhouse et Sud Alsace (GHRMSA)

blanc a été déclenché aux HCC le 11 mars, avec là aussi déprogrammation de toutes les activités.

Le 16 mars, les capacités de réanimation départementales étaient saturées, malgré l'ouverture de lits de réanimation supplémentaires dans les blocs opératoires, ainsi que des transferts vers des réanimations de départements limitrophes. Les structures privées colmariennes ont transformé leurs lits de soins continus et lits de réanimation, tout comme le centre hospitalier de Sélestat, situé dans le Bas-Rhin mais appartenant au même GHT que Colmar. À partir du 17 mars, le Samu 68 a mis en place une cellule de coordination des transferts, en charge d'identifier dans chaque établissement les patients de réanimation présentant des critères de stabilité compatibles avec un transfert, puis des réanimations en capacité de les accueillir et un vecteur adapté. Ainsi et jusqu'au 9 avril, un total de 330 patients ont été transférés à partir du Haut-Rhin, dont 60 à l'étranger. Cette rotation libérant des places de réanimation a permis de toujours pouvoir accueillir les nouveaux patients intubés dans nos services.

Le 23 mars, le GHRMSA a ouvert un deuxième SU permettant une séparation totale des flux Covid et non Covid. Le lendemain, l'élément mobile de réanimation du Service 
de santé des armées (EMR-SSA) installé sur un parking du GHRMSA a ouvert sa structure de réanimation progressivement, permettant d'accueillir jusqu'à 30 patients de réanimation.

\section{Organisation}

\section{Accueil et triage}

Au niveau de l'HCC, la configuration des locaux a rapidement permis d'identifier dans le secteur médecine un circuit dédié à chaque catégorie de patients, les circuits patients valides/patients en brancards devenant respectivement non Covid/Covid avec accès direct vers l'UHCD. L'UHCD de 15 lits a été renommée zone Covid destinée à accueillir toutes les suspicions dans l'attente de leur qualification biologique et/ou scanographique. Très rapidement, le 9 mars, soit cinq jours après le premier cas, le secteur médecine a été annexé par l'UHCD pour étendre la zone d'accueil Covid, laissant seul le secteur de traumatologie pour l'ensemble des patients non Covid, avec accès différenciés et flux vers les plateaux techniques également distincts.

Au niveau du GHRMSA, deux zones ont été délimitées au sein du SU avec un poste IOA dédié afin d'organiser des flux distincts Covid/non Covid. Le dimensionnement de ces zones a évolué avec l'augmentation du nombre de cas Covid. Un tri a été établi en amont pour orienter les patients vers les deux postes de tri IOA. Malgré les efforts réalisés, la configuration des locaux n'a pas permis une étanchéité entre les deux secteurs, et certains personnels sont passés régulièrement de l'un à l'autre avec des tenues inadaptées. Cela nous a amenés dans un second temps à ouvrir un second service d'urgence au sein du secteur de consultation de traumatologie pour y accueillir les patients non Covid. Un pré-tri a alors été organisé par le SAMU-Centre 15 et des secouristes de la Croix-Rouge munis d'un questionnaire type pour orienter les vecteurs vers l'un ou l'autre de ces SU.

\section{Stratégie d'orientation post-urgence}

Au sein des HCC, l'UHCD a été la première zone Covid du fait de sa configuration, avec neuf chambres seules disposant d'un système de remplacement de l'air performant, possibilité d'habillage et déshabillage à l'extérieur des chambres, possibilité de nettoyage efficace des locaux. Les indications conventionnelles d'UHCD ont été prises en charge soit en hospitalisations conventionnelles, soit directement en secteur de psychiatrie. Cependant, du fait de l'évolution, il a fallu rapidement étendre cette zone, ce qui a pu se faire tout naturellement en raison de la chute d'activité des patients non Covid ( $-30 \%$ en avril, $-50 \%$ en mai versus 2019$)$. Les patients suspects de Covid y étaient hospitalisés dans l'attente des résultats des examens complémentaires et d'être orientés dans une unité d'hospitalisation adaptée ; ce fonctionnement n'a tenu qu'une semaine, laissant le temps au reste de l'établissement de monter en charge ses secteurs Covid en hospitalisation conventionnelle. Dès la seconde semaine, UHCD et secteur de médecine, soit 26 places, ont fonctionné en permanence à l'accueil des patients Covid-19, avec réalisation des prélèvements viraux et orientation vers les zones Covid ou non Covid. L'objectif affiché était d'hospitaliser en zone Covid uniquement les cas confirmés. L'arrivée des tests rapides réalisés sur site a été un apport considérable dans la gestion des flux. Notre pic d'activité est survenu le 23 mars avec 64 patients Covid-19 positifs dans la journée.

Au sein du GHRMSA, les patients suspects Covid et présentant des critères d'hospitalisation ont été directement orientés dans des unités Covid, où ils bénéficiaient de la réalisation des prélèvements viraux avant éventuellement d'être réorientés selon les résultats. Devant le flux de patients et la gestion des patients graves aux urgences, cette organisation a permis une meilleure fluidité et répartition des charges entre SU et unité de soins. Ce n'est qu'à partir de fin mars, lorsque les tableaux cliniques sont devenus moins typiques, que les tests RT-PCR rapides ont été disponibles et que ces tests ont été réalisés aux urgences avant orientation.

\section{Prise en charge des patients en détresse respiratoire}

Les stratégies de prise en charge des patients en détresse respiratoire ont évolué en fonction des avancées scientifiques et des différentes recommandations [3-5]. Les tout premiers cas de détresse respiratoire ont été déstabilisants : $\mathrm{SpO}_{2}$ à $60 \%$ sans polypnée, sans tirage. Une attitude attentiste a été proposée avec oxygénation à fort débit, puis rapidement contredite par une potentielle surexposition des soignants par les effets d'aérosolisation, conduisant à une prise en charge systématique par intubation après sédation. Très rapidement, ces patients se sont succédés aux urgences, et le nombre d'intubations orotrachéales de à 2 quotidiennes en situation normale augmentera jusqu'à 15 aux HCC et 20 au GHRMSA. En six semaines, nous avons revu plus de fois nos protocoles d'intubation qu'au cours des cinq dernières années ; BAVU à usage unique pour tout le monde, acquisition de vidéolaryngoscopes et formation pratique rapide de tous les opérateurs, protocoles de sédation. A posteriori, et au vu des études actuellement disponibles, il est plus que probable que cette prise en charge systématique par intubation trachéale aurait pu être évitée notamment en faveur de l'oxygénothérapie haut débit $[6,7]$.

\section{Adaptation du système de santé}

Afin de limiter la sollicitation des SU, et d'optimiser la prise en charge des patients, des adaptations ont été mises en place 
avec l'ensemble des acteurs de santé du département. La ML a répondu présente massivement, n'adressant plus de patient hors Covid sur les SU, sauf urgence médicale réelle. Les établissements de santé privés se sont rapidement coordonnés avec le secteur public, en transformant des lits d'USC en lits de réanimation lourde et en mettant des personnels (IADE et anesthésistes) à disposition des réanimations publiques ; des autorisations temporaires d'activité de réanimation ont été accordées par l'agence régionale de santé à ces structures. Les médecins coordinateurs des établissements d'hébergement pour personnes âgées dépendantes (EHPAD) ont joué un rôle majeur de régulation, n'évacuant plus leurs patients vers le centre hospitalier de référence et assurant les soins localement dans leur structure, y compris pour les patients oxygénodépendants ou en fin de vie. La régulation médicale a joué un rôle important, d'une part en assurant le rôle de conseil auprès des patients présentant des tableaux symptomatiques simples, d'autre part en limitant les recours aux SU aux seuls patients présentant des critères de mauvaise tolérance et enfin en mettant en place des dispositifs de visite à domicile ou de consultation y compris en dehors des horaires de permanence des soins (partenariat avec SOS Médecins, déploiement de la plateforme Entr'acte, ouverture d'une consultation de médecine générale assurée par des libéraux à l'entrée du GHRMSA), intégration d'une consultation de ML dans le cadre des SASPAS aux HCC. La collaboration avec le service départemental d'incendie et de secours (SDIS) 68 a été exemplaire sur plusieurs plans : retour de patients pris en charge en véhicule de secours d'assistance aux victimes (VSAV) à domicile, création d'une salle de délestage des appels SAMU-Centre 15 au sein du centre opérationnel départemental d'incendie et de secours, transport de patients lourds, médicalisation de transports longue distance, collaboration entre pharmacies hospitalières et du SDIS avec fourniture de matériels de protection, de médicaments, afin d'éviter les ruptures dans les centres hospitaliers ; mais surtout, mise en place à Colmar d'équipes préhospitalières médicalisées par les médecins du SDIS, d'évaluation clinique des patients suspects d'infection à
Covid-19, avec la possibilité, en cas d'absence de critères de gravité, de laisser les patients à domicile, relais pris par le médecin traitant.

\section{Gestion des lits Covid}

La gestion des lits Covid s'est faite selon trois plans principaux : les lits de réanimation, les lits d'hospitalisation conventionnelle et les SSR. Cette gestion a été centralisée par la cellule de crise, avec analyse pluriquotidienne.

Les HCC disposaient de 30 lits de réanimation autorisés, capacité rapidement dépassée d'autant que les taux d'occupation habituels approchent les $100 \%$. Les unités de soins continus attenantes à ces réanimations ont été armées en lits de réanimation, portant le chiffre à 40 lits, une salle de surveillance postinterventionnelle (SSPI) puis une seconde ont aussi été armées, portant le chiffre à 60 , et avec l'aide du secteur privé et du centre hospitalier de Sélestat situé à quelques kilomètres de Colmar (même GHT), la capacité maximale a pu être portée à 72 lits de réanimation, tous équipés en respirateurs lourds et en personnels qualifiés. À la fin avril, 328 patients ont été admis en réanimation, en sus des 134 patients transférés dans d'autres centres français ou étrangers (Tableau 1). En hospitalisation conventionnelle, un secteur dédié, initialement proposé de 30 lits, a rapidement dû être doublé, puis étendu à 90, 120, 180 lits, sans compter les cohortes dans certains services de spécialités. Tous nos partenaires habituels d'aval, public ou privé, ont assuré les parts de travail, avec ouverture de lits de soins de suite et réadaptation (SSR) Covid, et flux de sorties d'hospitalisation accélérés. Au total, 552 lits Covid ont été disponibles à l'acmé de la crise et ont accueilli près de 1700 patients.

Le GHRMSA disposait quant à lui habituellement de 36 lits de réanimation. Rapidement, la demande de réanimation devenant très importante, des salles de bloc opératoire puis les SSPI ont été transformées en lit de réanimation, permettant d'atteindre 68 lits de réanimation auxquels se sont ajoutés les 30 lits de l'EMR-SSA. Le GHRMSA a accueilli au total 353 patients dans ces lits, avec un taux de mortalité

Tableau 1 Évolution du capacitaire en lits de réanimation du Haut-Rhin (USC : unités de soins continus ; HCC : Hôpitaux civils de Colmar ; GHRMSA : Groupement hospitalier de la région de Mulhouse et Sud Alsace ; EMR-SSA : élément militaire de réanimation - Service de santé des armées)

\begin{tabular}{|lllll|}
\hline & $\begin{array}{l}\text { Lits de réanimation } \\
\text { conventionnels }\end{array}$ & $\begin{array}{l}\text { Lits créés } \\
\text { par transformation } \\
\text { d'USC }\end{array}$ & $\begin{array}{l}\text { Lits créés } \\
\text { par transformation } \\
\text { de blocs }\end{array}$ & $\begin{array}{l}\text { Création de lits } \\
\text { de réanimation }\end{array}$ \\
\hline HCC & 30 & 10 & 20 & \\
GHRMSA & 36 & - & 32 & 30 \\
Secteur privé & & 10 & & \\
EMR-SSA & & 10 & & \\
Sélestat & & & & \\
\hline
\end{tabular}


de $18,7 \%$. Au total, le département a disposé de 170 lits de réanimation pour 66 lits en temps normal. Dans les secteurs d'hospitalisation, le GHRMSA a créé progressivement des unités Covid qui totaliseront au plus haut 500 lits Covid dédiés où ont été accueillis 1921 patients.

\section{Renforts de personnels}

L'ensemble des activités programmées médicales et chirurgicales a été stoppé nette, permettant un redéploiement des personnels soit pour renfort sur les sites aigus, soit pour réaffectation des services et ouverture des lits Covid. Les secteurs des hôpitaux de jour et/ou de semaine ont été fermés ou transformés en unités d'hospitalisations conventionnelles. La majorité des services de spécialité, n'ayant quasiment plus d'activité propre durant les premières semaines, a proposé ses médecins disponibles pour renforcer les postes actifs, à savoir les urgences ou le centre de dépistage. Au-delà des redéploiements internes, nos établissements ont eu recours au rappel de personnels retraités, à l'embauche de personnels intérimaires, au recours à la réserve sanitaire et à l'accueil de nombreux personnels de santé affluant de plusieurs régions de France non soumises à l'épidémie pour prêter main-forte au sein de nos unités. Ainsi pour le GHRMSA, cela a représenté au total 400 personnels non médicaux et 185 médecins qui sont venus renforcer ponctuellement les effectifs de l'établissement. Les HCC quant à eux n'ont eu droit qu'à 13 paramédicaux et 5 médecins ! C'est cet incomparable effet de solidarité de tous les acteurs du secteur hospitalier, soignants, direction, non-soignants, et de ces renforts externes qui a permis l'adaptation rapide et réactive de nos établissements.

\section{Discussion}

Malgré l'anticipation des HCC, malgré les dispositifs mis en place, malgré la formation des IOA et paramédicaux, malgré une zone d'accueil dédiée Covid en UHCD, le premier patient n'avait aucun lien avec Wuhan ou la Chine, n'avait pas voyagé, n'avait pas de signe respiratoire à l'admission, n'avait pas de température, n'avait pas de contact potentiel. Il s'agissait d'un patient admis en préhospitalier pour un accident de la voie publique le 4 mars, véhicule seul en cause, pris en charge par le SDIS. Il fut installé en secteur de traumatologie, a été vu par l'équipe en place, a été adressé en radiologie pour des clichés standard et, au retour aux urgences, a toussé, raison pour laquelle un interrogatoire a été fait à nouveau, mettant en évidence des frissons, une sortie de route liée à cet état, et la RT-PCR est revenue positive 48 heures plus tard, après avoir été en contact sans protection avec plus de 20 soignants.

L'organisation des deux SU a été sensiblement différente, mais respectant la division des flux Covid/non
Covid. Elle était d'autant plus pertinente à Colmar que les équipes sont entraînées aux procédures d'habillage/déshabillage et que les locaux sont adaptés à la division des flux. Mais l'évolution fréquente des recommandations et des organisations a compliqué l'adaptation des personnels. Les locaux doivent permettre l'étanchéité des zones évitant le passage de personnels d'une zone à l'autre. L'orientation des flux d'hospitalisation post-urgence s'est appuyée sur deux modalités différentes qui ont répondu à des organisations validées par les établissements et ont permis de limiter le risque de diffusion de l'infection, avec un secteur tampon permettant d'attendre les résultats virologiques et d'imagerie classant les patients.

Malgré les stocks de nos établissements notamment liés à l'activité NRBCe, la visibilité sur les EPI (FFP2, charlottes, tenues de protection, surblouses...), voire en solutés hydroalcooliques (SHA), a à peine permis de faire face aux dix premiers jours de fonctionnement. Les services logistiques ont fait un travail remarquable, mais rapidement limité par les réseaux habituels, tous les fabricants s'annonçant en rupture de stock simultanément, et tous les fabricants italiens, allemands ou chinois ne pouvant plus exporter. Il a fallu trouver des alternatives à nos circuits habituels d'approvisionnement pour éviter toute pénurie ou rupture : visite des bureaux de vote du premier tour des élections municipales pour récupérer les masques et SHA fournis, appel de l'ensemble des métiers de la bouche et des vignerons pour récupérer les tenues, appel direct aux secteurs sécurité de grandes entreprises. Cette utilisation massive n'a pu être anticipée du fait de l'intensité de progression de la pandémie au niveau local, le nombre de patients atteints doublant toutes les 36 à 48 heures. Les stocks d'État n'ont été débloqués qu'après une quinzaine de jours.

Avec nos premiers cas positifs, les 2 et 4 mars sur Mulhouse et Colmar, nos centres ont été sursaturés en à peine une semaine. Malgré les échanges avec la préfecture et l'agence régionale de santé, aucune mesure spécifique n'a été prise pour renforcer nos moyens en ressources matérielles ou humaines. Les préconisations nationales tant en termes de moyens de protection que de stratégie de dépistage ou de tests ont rapidement été inapplicables dans le HautRhin. Le discours national était en total décalage avec l'ampleur de ce que nous vivions. Nous avons alors joué notre rôle de lanceur d'alerte par la diffusion d'un message d'alerte adressé aux référents nationaux ministériels et professionnels (Société française de médecine d'urgence [SFMU] et Samu et Urgences de France [8]). Il s'agissait de permettre à nos collègues d'autres établissements de pouvoir se préparer à l'ampleur de ce qui allait leur arriver, sachant qu'une certaine inertie de progression de la vague avait été décrite. Colmar avait 24 à 36 heures de retard par rapport à Mulhouse, et Strasbourg 24 à 36 heures de retard par rapport à Colmar. Ce message d'alerte a été reçu par le 
terrain comme informatif et a permis un degré certain de préparation pour les centres suivants. Force est de constater qu'une fois de plus nos ministères ont eu des difficultés à percevoir la gravité de notre situation, tant que la région parisienne n'a pas été atteinte.

Les principales difficultés ont résidé dans le retard de prise en compte de la gravité de la situation locale par les instances nationales, avec pour première conséquence de grandes difficultés d'approvisionnement sur les EPI ; dans l'insuffisance du nombre de lits de réanimation nécessitant la création de lits supplémentaires puis le recours massif aux transferts de patients réanimatoires stables vers d'autres réanimations pour laisser des places aux patients en détresse arrivant en continu ; les renforts en ressources humaines rendus nécessaires par les nouvelles organisations et le remplacement des personnels infectés.

Les difficultés de gestion de cette crise ont été multiples : virus inconnu, ampleur dévastatrice, intensité de survenue, importance des flux en continu de patients lourds. Situation inédite pour tous, cette pandémie doit faire réfléchir :

- au maintien d'une flexibilité des ressources humaines, flexibilité locale mais également nationale afin de renforcer des équipes en difficulté ; c'est en partie le rôle des groupements hospitaliers de territoire (GHT), mais ce dimensionnement reste insuffisant avec ce type de crise ;

- au maintien de stocks de matériels nécessaires sur site afin d'équiper des lits conventionnels et permettre leur bascule en lits de soins critiques ; la principale problématique restera le coût d'immobilisation, mais aussi la gestion de ce stock et sa maintenance ;

- au maintien de stocks en quantités suffisantes en EPI sur chaque site afin de permettre une protection optimale de nos soignants et en garantissant au moins six à huit semaines d'autonomie en utilisation intensive; reste à voir la possibilité d'effectuer actuellement cette remise en stock, face aux difficultés d'approvisionnement, aux principes de gestion en flux tendus des fournisseurs, aux manques de matières premières et à la circulation moindre des marchandises ;

- à ajouter cette nouvelle contrainte dans les restructurations architecturales : multiplier les possibilités de cohabitation de flux de patients, tout en conservant des possibilités d'isolement et sans démultiplier les zones techniques ; accès distincts, chemins critiques du patient distincts, sor- ties distinctes de deux, voire trois typologies de patients sont à mettre en place ;

- à la limitation des patients adressés dans le cadre d'une pandémie au centre hospitalier; le SAMU-Centre 15 joue un rôle primordial, mais ne peut dans tous les cas juger de la nécessité d'une hospitalisation ; la mise en place, en sus des équipages Smur d'équipes d'évaluation préhospitalière clinique peut se justifier, avec implication de la ML ; une évaluation avec prélèvement de type RT-PCR (ou équivalent en cas d'un autre agent pathogène suspecté) à domicile pourra être effectuée, relais pris pour les résultats et pour une réévaluation à 24 ou 36 heures par le médecin traitant ; en cas d'aggravation clinique, une hospitalisation sans passage par les SU doit être possible, et en cas d'amélioration, le maintien à domicile se poursuit ;

- à l'optimisation de la communication entre acteurs de terrain, afin de permettre une meilleure réactivité ;

- à la clarification de l'action du niveau régional de coordination de crise ; coordination qui a fait preuve d'une méconnaissance des problématiques de terrain, d'une immaturité à piloter une crise, usant d'ordres et de contre-ordres ou de décisions inopérantes.

\section{Conclusion}

Les HCC et le GHRMSA ont été confrontés à une crise sanitaire sans précédent liée au développement fulgurant de l'épidémie de Covid-19, alors que la France ne connaissait que quelques clusters isolés. La violence de son développement n'a pas permis aux établissements d'anticiper leur organisation. Pour autant, ils ont fait face à cet afflux de patients au prix de réorganisations profondes, d'un déclenchement de plan blanc avec arrêt de toutes les organisations programmées, d'une adaptation de l'accueil et du tri des établissements s'appuyant sur des circuits distincts, et grâce à l'implication de tous. La cohésion intrahospitalière majeure entre soignants, direction et tous les acteurs de terrain ainsi que l'élan de solidarité exceptionnel ont permis l'adaptation et la flexibilité de nos hôpitaux nécessaires pour accueillir ce flux dont aucun plan n'avait permis l'anticipation.

Liens d'intérêts : les auteurs déclarent ne pas avoir de liens d'intérêts. 


\section{Éléments remarquables}

- Un soutien ferme et indéfectible de nos directions

- Une solidarité spontanée régionale et nationale de terrain

-Une mobilisation massive des personnels soignants

- Un travail coordonné des services de santé publics et privés

- Une adaptabilité exceptionnelle de nos unités et établissements

- Une maladie mortelle qui s'évite en se lavant les mains

et avec des gestes barrières

\section{Postes d'améliorations}

- Une absence de connaissance de terrain des hautes instances

- Une inertie de décisions politiques incompatibles avec une gestion de crise de ce type

- Un ennemi inconnu il y a encore quelques mois qui ne nous

a pas encore tout dit

- Développer au sein de l'ARS une compétence de gestion de crise (disponibilité des lits centralisée, logistique de transfert, coordination des moyens, recommandations,

approvisionnements...)

- Des outils d'information et de coordination régionaux

à développer

- Penser à se laver les mains, savoir éternuer, se moucher : il faut apprendre l'hygiène aux Français!

\section{Références}

1. Adhanom Ghebreyesus T (2020) Allocution liminaire du point presse sur la Covid-19 du 24 février 2020. https://www.who.int/ $\mathrm{fr} / \mathrm{dg} /$ speeches/detail/who-director-general-s-opening-remarks-atthe-media-briefing-on-covid-19-24-february-2020 (Dernier accès le 18 juillet 2020)

2. Salomon J (2020) Point presse du ministère de la Santé, mardi 3 mars 2020. https://solidarites-sante.gouv.fr/soins-et-maladies/maladies/maladies-infectieuses/coronavirus/etat-des-lieux-et-actualites/ article/points-de-situation-janvier-fevrier-mars-2020\#Point-dumardi-3-mars-2020 (Dernier accès le 30 juillet 2020)

3. Haute Autorité de santé (2020) Recommandations Covid-19. https://www.has-sante.fr/jcms/p_3168585/fr/tous-les-travaux-de-lahas-covid-19 (Dernier accès le 18 juillet 2020)

4. Coordination opérationnelle risque épidémique et biologique (COREB) (2020) Référentiels et procédures. https://www.coreb. infectiologie.com/fr/referentiels-procedures.html (Dernier accès le 18 juillet 2020)

5. Santé publique France (2020) Outils pour les professionnels de santé. https://www.santepubliquefrance.fr/dossiers/coronavirus-covid19/covid-19-outils-pour-les-professionnels-de-sante (Dernier accès le 18 juillet 2020)

6. Whittle JS, Pavlov I, Sacchetti AD, et al (2020) Respiratory support for adult patients with Covid-19. J Am Coll Emerg Physicians Open 1 (in press)

7. Coppo A, Bellani G, Winterton D, et al (2020) Feasibility and physiological effects of prone positioning in non-intubated patients with acute respiratory failure due to Covid-19 (PRON-Covid): a prospective cohort study. Lancet Respir Med 8:765-74

8. Braun F (2020) Une crise sanitaire avant tout. Ann Fr Med Urgence 10:199-201 